Nova Southeastern University

\title{
The Late Stellar Assembly of Massive Cluster Galaxies via Major Merging
}

\author{
Kim-Vy H. Tran \\ University of Zürich \\ John Moustakas \\ New York University \\ Anthony H. Gonzalez \\ University of Florida \\ Lei Bai \\ University of Arizona \\ Dennis Zaritsky \\ University of Arizona \\ See next page for additional authors
}

Follow this and additional works at: https://nsuworks.nova.edu/cnso_chemphys_facarticles Part of the External Galaxies Commons

\section{NSUWorks Citation}

Tran, K. H., Moustakas, J., Gonzalez, A. H., Bai, L., Zaritsky, D., \& Kautsch, S. (2008). The Late Stellar Assembly of Massive Cluster Galaxies via Major Merging. Astrophysical Journal Letters, 683, (1), L17 - L20. https://doi.org/10.1086/591422. Retrieved from https://nsuworks.nova.edu/cnso_chemphys_facarticles/111 
Authors

Kim-Vy H. Tran, John Moustakas, Anthony H. Gonzalez, Lei Bai, Dennis Zaritsky, and Stefan Kautsch 


\title{
THE LATE STELLAR ASSEMBLY OF MASSIVE CLUSTER GALAXIES VIA MAJOR MERGING
}

\author{
Kim-Vy H. Tran, ${ }^{1}$ John Moustakas, ${ }^{2}$ Anthony H. Gonzalez, ${ }^{3}$ Lei Bai, ${ }^{4}$ Dennis Zaritsky, ${ }^{4}$ and Stefan J. Kautsch ${ }^{3}$ \\ Received 2008 June 4; accepted 2008 June 26; published 2008 July 22
}

\begin{abstract}
We present multiwavelength observations of the brightest galaxies in four X-ray-luminous groups at $z \sim$ 0.37 that will merge to form a cluster comparable in mass to Coma. Ordered by increasing stellar mass, the four brightest group galaxies (BGGs) present a time sequence where BGG-1, 2, and 3 are in merging systems and BGG-4 is a massive remnant $\left(M_{*}=6.7 \times 10^{11} M_{\odot}\right)$. BGG-1 and 2 have bright, gravitationally bound companions and BGG-3 has two nuclei separated by only $2.5 \mathrm{kpc}$; thus, merging at $z<0.5$ increases the BGG mass by $\gtrsim 40 \%$ $\left(t_{\mathrm{MGR}}<2 \mathrm{Gyr}\right)$ and $V$-band luminosity by $\sim 0.4 \mathrm{mag}$. The BGGs' rest-frame $(B-V)$ colors correspond to stellar ages of $>3 \mathrm{Gyr}$, and their tight scatter in $(B-V)$ color $\left(\sigma_{B V}=0.032\right)$ confirms that they formed the bulk of their stars at $z>0.9$. Optical spectroscopy shows no signs of recent $(<1.5 \mathrm{Gyr})$ or ongoing star formation. Only two BGGs are weakly detected at $24 \mu \mathrm{m}$, and X-ray and optical data indicate that the emission in BGG-2 is due to an AGN. All four BGGs and their companions are early-type (bulge-dominated) galaxies, and they are embedded in diffuse stellar envelopes up to $\sim 140 \mathrm{kpc}$ across. The four BGG systems must evolve into the massive, red, early-type galaxies dominating local clusters. Our results show that (1) massive galaxies in groups and clusters form via dissipationless merging and (2) the group environment is critical for this process.
\end{abstract}

Subject headings: galaxies: clusters: general — galaxies: elliptical and lenticular, $\mathrm{cD}$ — galaxies: evolution

Online material: color figure

\section{INTRODUCTION}

Whether massive cluster galaxies at $z \sim 0$ build up a significant fraction of their stellar masses at $z<1$ via merging continues to be an intensely debated question. In the paradigm of hierarchical formation, galaxies continue to grow via accretion of smaller satellites (Peebles 1970), and recent models predict that brightest cluster galaxies assemble half their stellar masses at $z<0.5$ (De Lucia \& Blaizot 2007). However, direct merging is not favored in virialized clusters due to their high velocity dispersions $\left(\sigma_{1 \mathrm{D}} \sim 1000 \mathrm{~km} \mathrm{~s}^{-1}\right)$.

Brightest cluster galaxies (BCG) are particularly compelling tracers of mass growth because they are the most massive galaxies in the universe, can be observed to high redshifts (Gunn \& Oke 1975), and should have a rich merger history. A number of BCGs in the local universe have multiple nuclei (Hoessel et al. 1985; Lauer 1986; Laine et al. 2003), which suggests recent merging. However, studies of BCGs in the more distant universe disagree as to whether BCGs have grown significantly in stellar mass since redshift $z \sim 1$ (Brough et al. 2002; Whiley et al. 2008).

While merging of red, luminous members is observed in groups and dynamically young clusters (van Dokkum et al. 1999; Tran et al. 2005b; McIntosh et al. 2007), the merging frequency and thus its importance remains uncertain. The key may be environment: merging is rare in established clusters but more frequent in groups that are accreted onto clusters. Because groups have a higher fraction of passive early-type galaxies relative to the field (Zabludoff \& Mulchaey 1998; Wilman et al. 2005; Jeltema et al. 2007), merging between such systems will form massive, passive galaxies.

Our study focuses on the brightest galaxies in four X-ray-

\footnotetext{
${ }^{1}$ Institute for Theoretical Physics, University of Zürich, CH-8057 Zürich, Switzerland.

${ }^{2}$ Center for Cosmology and Particle Physics, 4 Washington Place, New York University, New York, NY 10003.

${ }^{3}$ Department of Astronomy, University of Florida, Gainesville, FL 32611.

${ }^{4}$ Steward Observatory, University of Arizona, 933 North Cherry Avenue, Tucson, AZ 85721.
}

luminous galaxy groups at $z \sim 0.37$ discovered in the Las Campanas Distant Cluster Survey (LCDCS) ${ }^{5}$ (Gonzalez et al. 2001). These four galaxy groups are gravitationally bound to each other and form an extended structure that will assemble into a galaxy cluster comparable in mass to Coma by the present epoch (Gonzalez et al. 2005, hereafter G05). By studying galaxies in the group environment prior to cluster assembly, we directly test whether galaxy-galaxy merging on group scales can drive stellar growth in the massive galaxies observed in local clusters and whether a large fraction of new stars is formed in the process. Throughout the Letter, we use $H_{0}=70 \mathrm{~km} \mathrm{~s}^{-1}$ $\mathrm{Mpc}^{-1}, \Omega_{M}=0.3$, and $\Omega_{\Lambda}=0.7$; at $z=0.37$, this corresponds to a scale of $5.12 \mathrm{kpc} \operatorname{arcsec}^{-1}$ and a lookback time of $4 \mathrm{Gyr}$.

\section{OBSERVATIONS}

Follow-up Chandra ACIS-I imaging and optical spectroscopy of the unique supergroup 1120 field confirmed the existence of four X-ray-luminous groups at $z \sim 0.37$ (hereafter SG1120). These groups lie within a projected region of $\sim 3$ $\mathrm{Mpc}$ and have X-ray temperatures of $T_{\mathrm{X}}=1.7-3.0 \mathrm{keV}$ (G05). Our study combines imaging and spectroscopy from six different observatories. ${ }^{6}$

\footnotetext{
${ }^{5}$ The LCDCS was designed to find galaxy clusters at $z \sim 0.35-0.9$ and was sensitive to groups only at the lowest redshifts.

${ }^{6}$ This work is based on observations made with (1) the NASA/ESA Hubble Space Telescope, obtained at the Space Telescope Science Institute, which is operated by AURA, Inc., under NASA contract NAS 5-26555; (2) the Spitzer Space Telescope, which is operated by the Jet Propulsion Laboratory, California Institute of Technology under a contract with NASA; (3) the Chandra $X$-Ray Telescope, obtained at the Chandra X-Ray Observatory Center, which is operated by the Smithsonian Astrophysics Observatory for and on behalf of NASA under contract NAS8-03060; (4) the 4 m Mayall telescope at Kitt Peak National Observatory; (5) the $6.5 \mathrm{~m}$ Magellan Telescopes at Las Campanas Observatory, Chile; and (6) the ESO Telescopes at the Paranal Observatories (072.A-0367, 076.B-0362, and 078.B-0409).
} 
TABLE 1

Properties of Brightest Group Galaxies

\begin{tabular}{ccccccc}
\hline \hline BGG & $z$ & $V$ & $(B-V)$ & $\begin{array}{c}M_{*} \\
\left(M_{\odot}\right)\end{array}$ & $\begin{array}{c}L_{\mathrm{IR}}{ }^{\mathrm{a}} \\
\left(\mathrm{ergs} \mathrm{s}^{-1}\right)\end{array}$ & $\sigma_{\mathrm{ID}}{ }^{\mathrm{b}}\left(\mathrm{km} \mathrm{s}^{-1}\right)$ \\
\hline $1 \mathrm{a} \ldots \ldots$ & 0.3540 & -22.46 & 0.86 & $2.9 \times 10^{11}$ & $\ldots$ & $329 \pm 66$ \\
$1 \mathrm{~b} \ldots \ldots$ & 0.3532 & -21.49 & 0.85 & $1.2 \times 10^{11}$ & $\ldots$ & $329 \pm 66$ \\
$2 \mathrm{a} \ldots \ldots$ & 0.3706 & -22.97 & 0.94 & $5.0 \times 10^{11}$ & $1.5 \times 10^{44}$ & $421 \pm 83$ \\
$2 \mathrm{~b} \ldots \ldots$ & 0.3704 & -22.04 & 0.92 & $2.1 \times 10^{11}$ & $\ldots$ & $421 \pm 83$ \\
$3 \ldots \ldots$ & 0.3713 & -23.20 & 0.94 & $5.4 \times 10^{11}$ & $8.0 \times 10^{43}$ & $588 \pm 90$ \\
$4 \ldots \ldots$ & 0.3720 & -23.38 & 0.91 & $6.7 \times 10^{11}$ & $\ldots$ & $565 \pm 130$ \\
\hline
\end{tabular}

Note. - Positions for the individual galaxy groups are in G05.

${ }^{a}$ Infrared luminosities determined from SST/MIPS $24 \mu \mathrm{m}$ fluxes.

${ }^{\mathrm{b}}$ Line-of-sight velocity dispersions $\left(\sigma_{1 \mathrm{D}}\right)$ are measured using all members within $500 \mathrm{kpc}$ of the BGGs (13-36 galaxies for each group) and using the biweight estimator (Beers et al. 1990).

\subsection{Optical and Near-Infrared Imaging}

The optical photometry is measured from VLT/VIMOS imaging in $B V R$ for a nearly contiguous $18^{\prime} \times 20^{\prime}$ region and from a contiguous mosaic $\left(11^{\prime} \times 18^{\prime}\right)$ composed of 10 pointings taken with the HST/ACS in F814W. The high-resolution ACS imaging is critical for identifying close galaxy pairs and merger signatures. Two pointings with KPNO/FLAMINGOS also provide a contiguous $K_{s}$ mosaic $\left(16^{\prime} \times 19^{\prime}\right)$.

Using the HST/ACS mosaic as the master detection image, line-matched catalogs were generated using SExtractor version 2.5.0 (Bertin \& Arnouts 1996). Rest-frame absolute magnitudes, $K$-corrections, and stellar masses were determined with k-correct version 4.1 (Blanton \& Roweis 2007), which uses templates based on models from Bruzual \& Charlot (2003, hereafter BC03). We used $\mathrm{MAG}_{-}$AUTO photometry from the $B V R K_{s}$ imaging and assumed minimum photometric uncertainties in each bandpass of $0.05 \mathrm{mag}$. The photometry have also been corrected for foreground Galactic extinction using the Schlegel et al. (1998) dust maps and the O'Donnell (1994) Milky Way extinction curve, assuming $R_{V}=3.1$.

\subsection{Optical Spectroscopy}

The initial survey using VLT/VIMOS confirmed that four of the five X-ray regions are galaxy groups at $z \sim 0.37$ while the fifth is a cluster at $z=0.48$ (G05). Targets were selected from a magnitude-limited catalog $(R \leq 22.5 \mathrm{mag})$ and the spectra reduced using a combination of $\mathrm{IRAF}^{7}$ routines and custom software; see Tran et al. (2005a) for details on the reduction and redshift determination.

Further spectroscopy was obtained using Magellan/LDSS3 and VLT/FORS2. In both cases, targets were selected from $K_{s}$ catalogs $\left(K_{s} \leq 20 \mathrm{mag}\right)$; for reference, the brightest supergroup galaxy has $K_{s}=14.62 \mathrm{mag}$. Guided by the redshift distribution, we define the supergroup's redshift range to be $0.32 \leq z \leq 0.39$, where the brightest group galaxies lie at $0.354 \leq z \leq 0.372$ (Table 1). These spectra provided 97 new members for a total of 198 confirmed supergroup galaxies across the approximately $20^{\prime} \times 20^{\prime}$ region.

The medium-resolution spectroscopy corresponds to $0.7 \AA$ pixel $^{-1}$ (LDSS3), $1.65 \AA$ pixel $^{-1}$ (FORS2), and $2.5 \AA$ pixel $^{-1}$ (VIMOS). The spectral range for all three instruments covers [O II] $\lambda 3727$ to [O III] $\lambda 5007$ for most supergroup members.

\section{3. $24 \mu \mathrm{m}$ Imaging}

Wide-field MIPS imaging $\left(0.36^{\circ} \times 1^{\circ}\right)$ was taken in slowscan mode and the data processed with the MIPS Data Analysis

${ }^{7}$ IRAF is distributed by the National Optical Astronomy Observatory.

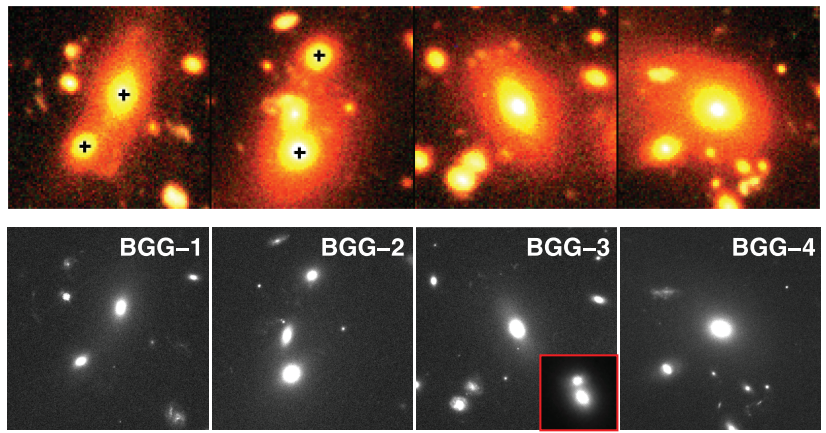

FIG. 1.-Top: VLT/VIMOS $B V R$ images $\left(20^{\prime \prime} \times 20^{\prime \prime}\right)$ of the four brightest group galaxies ordered in increasing stellar mass, i.e., a rough time sequence. BGG-1 and 2 both have bright, gravitationally bound companions (marked with crosses). All four BGGs have extended, diffuse stellar envelopes that are up to $140 \mathrm{kpc}$ across (BGG-1). Bottom: ACS F814W imaging of the same BGGs reveals the spectacular double nucleus in BGG-3 (image inset; projected separation of $2.5 \mathrm{kpc}$ ).

Tool (DAT ver. 3.02; Gordon et al. 2005), where array-averaged background subtraction was applied to improve the flat-fielding. At $24 \mu \mathrm{m}$, the exposure time is $\sim 1000 \mathrm{~s} \mathrm{pixel}^{-1}$ and the $3 \sigma$ point source detection limit $\sim 100 \mu \mathrm{Jy}$. We use DAOPHOT II (Stetson 1987) to detect sources and measure their fluxes, and we follow the same strategy as described in Papovich et al. (2004). The spectroscopically confirmed members are correlated with the $24 \mu \mathrm{m}$ catalog using a $2^{\prime \prime}$ search radius. Total IR luminosities are determined using star-forming galaxy templates developed by Dale \& Helou (2002) and $L_{\mathrm{IR}}$ converted to star formation rates using equation (3) in Kennicutt (1998).

\section{RESULTS}

\subsection{Brightest Group Galaxies}

Images of the brightest group galaxy (BGG) within each of the four X-ray-luminous groups at $z \sim 0.37$ are shown in Figure 1. The BGGs are ordered in increasing stellar mass (see $\S 2.1$ ) and are in the range $(2.9-6.7) \times 10^{11} M_{\odot}$ (Table 1).

All of the BGGs are early-type galaxies with no visual signs of ongoing star formation. These BGGs are in groups with masses less than that of galaxy clusters $\left(\sim 10^{14} M_{\odot}\right.$ vs. $\sim 10^{15}$ $M_{\odot}$ ) at $\sim 1 / 3$ the lookback time, yet they already morphologically resemble the massive early-type galaxies that are common in $z \sim 0$ clusters, e.g., Coma (Davis \& Geller 1976). The BGGs are even comparable in stellar mass to the most massive brightest cluster galaxies in the local universe $\left(M_{*} \sim 5 \times\right.$ $10^{11} M_{\odot}$; von der Linden et al. 2007)

Spectroscopy uncovers the second striking result: the two lowest mass BGGs are part of merging systems (Fig. 1). BGG-1 has a companion galaxy $\left(D_{\text {proj }}=34 \mathrm{kpc}, \delta v=170 \mathrm{~km} \mathrm{~s}^{-1}\right)$ where both are embedded in a common, extended envelope that has a semimajor axis of $\sim 140 \mathrm{kpc}$; this stellar envelope is especially prominent in the $R$-band imaging and is reminiscent of the stellar plume associated with a major merger forming a brightest cluster galaxy at $z=0.39$ (Rines et al. 2007). BGG-2 also has a companion galaxy $\left(D_{\text {proj }}=48 \mathrm{kpc}, \delta v=53 \mathrm{~km} \mathrm{~s}^{-1}\right.$; note $\delta v \approx \sigma_{c z}$ ) within a faint, extended stellar halo.

In the ground-based imaging (PSF $\sim 0.7^{\prime \prime}$ ), BGG-3 looks like a normal early-type galaxy, but the high-resolution ACS imaging $\left(0.05^{\prime \prime}\right.$ pixel $\left.^{-1}\right)$ reveals its spectacular double nucleus (Fig. 1). As part of our spectroscopic survey, we aligned a slit along the two nuclei and confirm that both are part of the same 


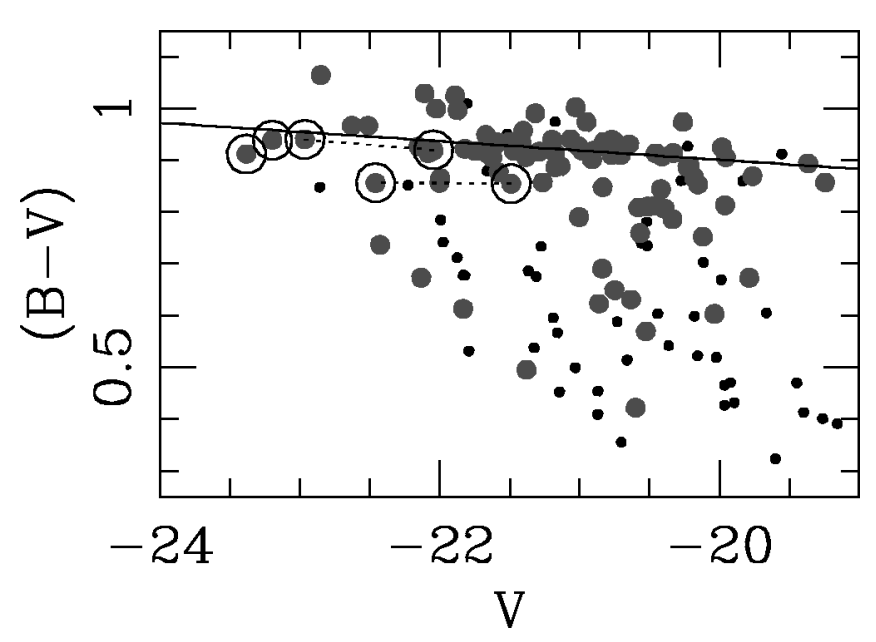

FIG. 2.-Rest-frame color-magnitude diagram for the 146 members $(0.32 \leq z \leq 0.39)$ on the ACS mosaic (small dots). The large filled dots are passive members ([O II] EW $<5 \AA$ ) and the large open circles denote the BGGs and merging companions (connected with the dotted lines). The slope of the CM relation is taken from CL 1358+62 $(z=0.33$; van Dokkum et al. 1998 ), and the fit is normalized to the passive members. After merging, the two faintest BGGs will increase their $V$-band luminosities by $\sim 0.4$ mag. [See the electronic edition of the Journal for a color version of this figure.]

galaxy. The nuclei are separated by only $2.5 \mathrm{kpc}$. BGG-3 also has a diffuse stellar halo.

BGG-4 is the most massive galaxy in SG1120 and lies in the group with the highest X-ray temperature (G05). Other than a diffuse stellar halo, BGG-4 has no obvious signs of recent $(t<1$ Gyr) merging, e.g., multiple nuclei. However, recent simulations indicate that massive ellipticals $\left(M_{*}>6 \times 10^{11} M_{\odot}\right)$ like BGG-4 form via dissipationless merging of two spheroids (Naab et al. 2006).

Taken as a whole, these four BGGs present a time sequence where luminous $\left(L>L^{*}\right)$ galaxies continue to merge at late times, as predicted by hierarchical formation (Peebles 1970). The merging galaxies are spheroids that have no visible signs of current or recent star formation but do have extended stellar envelopes. During the merging process, stars from these spheroids are gravitationally disrupted and likely to contribute to the intracluster light (Mihos 2003). The resulting merger remnant will be a massive, early-type galaxy embedded in an extended, diffuse stellar envelope, e.g., BGG-4.

\subsection{Color-Magnitude Diagram and Stellar Mass}

Figure 2 shows the rest-frame $(B-V)$ color versus absolute $V$-band magnitude for the 146 supergroup members that fall on the HST/ACS mosaic. Passive members $(N=88 ; \sim 60 \%)$, are well fitted using the color-magnitude (CM) relation measured in CL $1358+62$, a massive galaxy cluster at $z=0.33$ (van Dokkum et al. 1998, hereafter vD98). The CM relation is normalized to the passive members where outliers have been iteratively removed using the median absolute deviation. We note that $\sim 60 \%$ of the members are E/S0 galaxies.

All of the BGGs lie within $\Delta(B-V)>-0.1$ of the CM relation and are thus red. While they all lie blueward of the $\mathrm{CM}$ relation, assuming the BGGs evolve passively their $(B-V)$ colors will increase such that the BGGs will be tightly clustered around the CM relation by $z \sim 0$. Their $(B-V)$ colors already correspond to single stellar populations that are at least 3 Gyr old (solar metallicity; BC03).

Included in Figure 2 and Table 1 are the companion galaxies to BGG-1 and BGG-2. Once these companions merge with

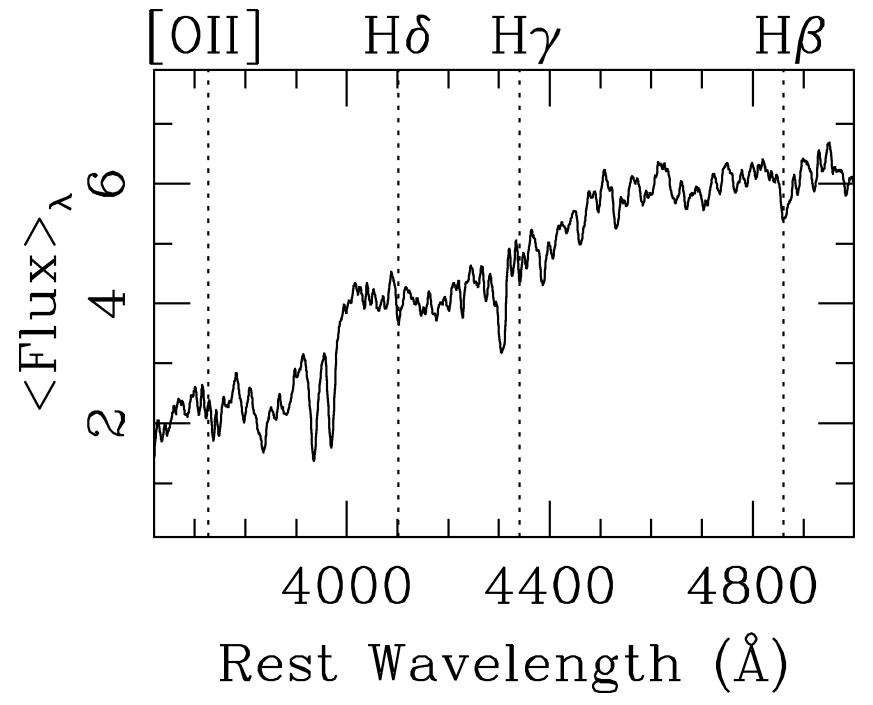

FIG. 3.-Composite rest-frame spectrum of the six members making up the merging BGG systems; each spectrum was normalized to its flux level at $6400<\lambda<6600 \AA$ before summing. The high signal-to-noise spectrum shows no signs of ongoing or recent star formation (within $\sim 1.5 \mathrm{Gyr}$ ).

their BGGs via, e.g., dynamical friction, the resulting BGGs will be $\sim 0.4$ mag brighter.

The stellar masses determined from the photometry provide further evidence of BGG buildup. The companion galaxies to BGG-1 and BGG-2 are each $~ 40 \%$ of their respective BGG's mass, and the two nuclei in BGG-3 have a luminosity ratio of about $1: 2$. Our data indicate that the BGGs grow in mass by $\geq 40 \%-50 \%$ during the most recent merger. We estimate the merging timescale by setting it equal to the dynamical friction timescale (Binney \& Tremaine 1987) and find that $t_{\mathrm{MGR}} \leqslant 2$ Gyr, consistent with merging timescales measured for luminous red galaxies (Conroy et al. 2007) and for close pairs in cosmological simulations (Kitzbichler \& White 2008).

A powerful feature of a color-magnitude analysis is using the scatter in color to determine the relative stellar ages of different galaxy populations (Bower et al. 1998). The $(B-V)$ scatter (RMS) for the six members in the BGG systems is 0.032; this is significantly smaller than the color scatter of the passive members $\left(\sigma_{B V}=0.116\right.$; Fig. 2$)$; i.e., the BGGs are more homogeneous in stellar age than the passive population.

Because $(B-V)$ scatter decreases as stellar populations age, we also can constrain the formation redshift of the BGGs. For simplicity, we model the BGGs as single stellar populations (SSPs) and allow SSPs to form any time up to $t$, where $t_{0}$ is the epoch at $z=0.37$. Using equation (A3) in vD98, we estimate the $(B-V)$ scatter from 5000 SSPs generated at random times and find that to have $\sigma_{B V}=0.032$, all of the SSPs must form by $t=0.65 t_{0}$. In the current cosmology, this corresponds to all of the stars in the BGGs forming at $z>0.9$.

Assuming the BGGs continue to evolve passively, their $(B-V)$ scatter decreases to $\sigma_{B V} \sim 0.020$ by $z=0$. This is even smaller than the intrinsic scatter of $\sigma_{B V}=0.028 \pm 0.004$ measured using the 104 brightest $H$-band early-type galaxies in Coma at $z=0.02$ (Eisenhardt et al. 2007). Note that $\sim 60 \%-$ $70 \%$ of the supergroup galaxies are already passive and/or red (Fig. 2); thus, later merging events are unlikely to significantly change the massive BGGs' mean stellar ages.

\subsection{Composite Optical Spectrum}

Figure 3 shows the composite rest-frame optical spectrum of the six galaxies in the BGG systems. The composite BGG 
spectrum has the strong $H$ and $K$ absorption features associated with an old ( $\left.>10^{9} \mathrm{Gyr}\right)$ stellar population and has no emission features nor Balmer absorption. Jeltema et al. (2007) also find that intermediate-redshift $(0.2<z<0.6)$ BGGs lack [O II] $\lambda 3727$ emission.

Optical emission lines are physically driven by ongoing star formation while strong Balmer absorption, $\mathrm{H} \delta$ in particular, provides a fossil record of any star formation that ended within the last 1.5 Gyr (e.g., Couch \& Sharples 1987). The absence of these features in the composite BGG spectrum indicates that these members have not formed any stars recently, and the spectrum's $D_{N}(4000)$ index of 1.72 corresponds to a mean stellar age of $\sim 1.7$ Gyr (solar metallicity; BC03).

\section{4. $24 \mu \mathrm{m}$ Detections}

Only BGG-2 and 3 are detected at $24 \mu \mathrm{m}$, and assuming the mid-infrared emission is due to dusty star formation leads to rates of 6.7 and $3.6 M_{\odot} \mathrm{yr}^{-1}$, respectively. However, dusty starforming red-sequence galaxies detected at $24 \mu \mathrm{m}$ tend to show weak [O II] $\lambda 3727$ emission in their optical spectra (Wolf et al. 2005), and neither BGG-2 nor 3 does (Fig. 3). While $24 \mu \mathrm{m}$ emission is often attributed to ongoing star formation, it can also be due to an active galactic nucleus (AGN). BGG-2 is an $\mathrm{X}$-ray point source; thus, both its X-ray and $24 \mu \mathrm{m}$ emission are likely due to an AGN. BGG-3's weaker $24 \mu \mathrm{m}$ detection makes it difficult to discriminate between a faint AGN and star formation.

BGG-2's multiwavelength properties are consistent with an old stellar population ( $t_{*} \gtrsim 2$ Gyr) and AGN. Current models favor the formation of luminous red galaxies via merging of progenitors with supermassive black holes; the gravitational perturbations bring fresh gas to the small central region and trigger an AGN phase (e.g., Springel et al. 2005). BGG-2, and possibly BGG-3, are compatible with such a model.

\section{DISCUSSION AND CONCLUSIONS}

These four BGG snapshots provide unprecedented observational evidence for the late $(z<0.5)$ stellar assembly of massive spheroidal galaxies in clusters via dissipationless merging of massive progenitors. Two of the four BGGs are part of merging pairs, and another has a double nucleus with a separation of only $2.5 \mathrm{kpc}$. When ordered by increasing stellar mass $\left[(2.9-6.7) \times 10^{11} M_{\odot}\right]$, the BGGs present a time sequence where galaxy-galaxy merging increases the BGG mass by $\gtrsim 40 \%\left(t_{\mathrm{MGR}} \lesssim 2 \mathrm{Gyr}\right)$ and $V$-band luminosity by $\sim 0.4 \mathrm{mag}$. Our results are remarkably consistent with recent claims from semianalytic models that brightest cluster galaxies tend to assemble at $z<0.5$ (De Lucia \& Blaizot 2007); such a model also naturally explains the observed number of brightest cluster galaxies in the local universe with multiple nuclei ( 20\%-40\%; Hoessel et al. 1985; Laine et al. 2003).

Despite the merger activity, the BGGs are dominated by old $\left(>10^{9}\right.$ Gyr) stellar populations and show little sign of ongoing or recent star formation. The BGGs have rest-frame $(B-V)$ colors corresponding to mean stellar ages of $>3 \mathrm{Gyr}$, and their tight scatter in color $\left(\sigma_{B V}=0.032\right)$ confirms that the BGGs formed their stars at $z>0.9$. If the BGGs continue to age passively, their color scatter by $z \sim 0$ will be even smaller than that of the $\sim 100$ brightest early-type galaxies in Coma $\left(\sigma_{B V}=\right.$ 0.020 vs. 0.028). Their composite optical spectrum shows no sign of star formation in the last $\sim 1.5$ Gyr. While BGG-2 and 3 are weakly detected at $24 \mu \mathrm{m}$, the X-ray and optical data indicate that BGG-2's $24 \mu \mathrm{m}$ emission is due to AGN rather than star formation, and possibly for BGG-3 as well.

SG1120 thus provides unique and powerful confirmation of hierarchical formation on both galaxy and cluster scales because (1) it has massive galaxies assembling from galaxy-galaxy merging and (2) it is a protocluster assembling from the merging of galaxy groups. Our findings show that the most massive galaxies in groups and clusters form via dissipationless merging and that the galaxy group environment is critical to forming the massive galaxies dominating local clusters.

K. T. acknowledges support from the Swiss National Science Foundation (grant PP002-110576) and thanks J. Blakeslee for help during the initial ACS reduction. J. M. acknowledges support from NASA-06-GALEX06-0030 and Spitzer G05-AR50443, and L. B. from NASA Spitzer programs through JPL subcontracts 1255094 and 1256318 . Support was also provided by NASA HST GO-10499, JPL/Caltech SST GO-20683, and Chandra GO2-3183X3.

\section{REFERENCES}

Beers, T. C., Flynn, K., \& Gebhardt, K. 1990, AJ, 100, 32

Bertin, E., \& Arnouts, S. 1996, A\&AS, 117, 393

Binney, J., \& Tremaine, S. 1987, Galactic Dynamics (Cambridge: Princeton Univ. Press)

Blanton, M. R., \& Roweis, S. 2007, AJ, 133, 734

Bower, R. G., Kodama, T., \& Terlevich, A. 1998, MNRAS, 299, 1193

Brough, S., et al. 2002, MNRAS, 329, L53

Bruzual, G., \& Charlot, S. 2003, MNRAS, 344, 1000 (BC03)

Conroy, C., Ho, S., \& White, M. 2007, MNRAS, 379, 1491

Couch, W. J., \& Sharples, R. M. 1987, MNRAS, 229, 423

Dale, D. A., \& Helou, G. 2002, ApJ, 576, 159

Davis, M., \& Geller, M. J. 1976, ApJ, 208, 13

De Lucia, G., \& Blaizot, J. 2007, MNRAS, 375, 2

Eisenhardt, P. R., et al. 2007, ApJS, 169, 225

Gonzalez, A. H., et al. 2001, ApJS, 137, 117 2005, ApJ, 624, L73 (G05)

Gordon, K. D., et al. 2005, PASP, 117, 503

Gunn, J. E., \& Oke, J. B. 1975, ApJ, 195, 255

Hoessel, J. G., Borne, K. D., \& Schneider, D. P. 1985, ApJ, 293, 94

Jeltema, T. E., et al. 2007, ApJ, 658, 865

Kennicutt, R. C., Jr. 1998, ApJ, 498, 541

Kitzbichler, M. G., \& White, S. D. M. 2008, preprint (arXiv:0804.1965)

Laine, S., van der Marel, R. P., Lauer, T. R., Postman, M., O’Dea, C. P., \& Owen, F. N. 2003, AJ, 125, 478
Lauer, T. R. 1986, ApJ, 311, 34

McIntosh, D. H., et al. 2007, preprint (arXiv:0710.2157)

Mihos, C. 2003, in Clusters of Galaxies: Probes of Cosmological Structure and Galaxy Evolution, ed. J. Mulchaey, A. Dressler, \& A. Oemler (Cambridge: Cambridge Univ. Press)

Naab, T., Khochfar, S., \& Burkert, A. 2006, ApJ, 636, L81

O'Donnell, J. E. 1994, ApJ, 422, 158

Papovich, C., et al. 2004, ApJS, 154, 70

Peebles, P. J. E. 1970, AJ, 75, 13

Rines, K., Finn, R., \& Vikhlinin, A. 2007, ApJ, 665, L9

Schlegel, D. J., Finkbeiner, D. P., \& Davis, M. 1998, ApJ, 500, 525

Springel, V., et al. 2005, Nature, 435, 629

Stetson, P. B. 1987, PASP, 99, 191

Tran, K. H., et al. 2005a, ApJ, 619, 134

Tran, K.-V. H., et al. 2005b, ApJ, 627, L25

van Dokkum, P. G., et al. 1998, ApJ, 500, 714 (vD98) 1999, ApJ, 520, L95

von der Linden, A., Best, P. N., Kauffmann, G., \& White, S. D. M. 2007, MNRAS, 379, 867

Whiley, I. M., et al. 2008, MNRAS, 387, 1253

Wilman, D. J., et al. 2005, MNRAS, 358, 71

Wolf, C., Gray, M. E., \& Meisenheimer, K. 2005, A\&A, 443, 435

Zabludoff, A. I., \& Mulchaey, J. S. 1998, ApJ, 496, 39 